\title{
"Tratamiento de la candidiasis vaginal con dosis única de fluconazol oral: estudio abierto, prospectivo, no comparativo"
}

\author{
Amparo Lucena de García'; Susy Manotas'; William Onatra²; Rodrigo Cifuentes ${ }^{3}$;
} Gabriel Acuña+; Agustín González ${ }^{5}$

\begin{abstract}
RESUMEN OBJETIVO: Evaluar la eficacia y seguridad de una dosis única de $150 \mathrm{mg}$ de fluconazol en el tratamiento de la candidiasis vaginal sintomática, en base a un diseño prospectivo, abierto, no comparativo, realizado en la consulta privada de los médicos paticipantes y consulta externa de PROFAMILIA en Cali, ORIENTAME y el Instituto Matero Infantil en Santafé de Bogotá, en pacientes ambulatorias sintomáticas, con diagnóstico clínico comprobado por frotis directo con KOH y cultivo positivos.
\end{abstract}

MATERIAL Y METODOS: No se permitió el empleo de otros antimicóticos tópicos o sistémicos concomitantemente. Al azar se entregó o no tratamiento para el compañero sexual estable de la paciente, resultando dos grupos que se controlaron una y cuatro semanas después de suministrada la dosis única.

ELEMENTOS A EVALUAR: 1. Negativización de los cultivos y desaparición del cuadro clínico. 2. Efecto que, sobre la evolución del cuadro clínico, pudiera producir el tratamiento al compañero sexual estable.

110 pacientes incluidas, pero 109 analizadas; una nunca retornó a los controles. Edad $30.29 \pm 6.6$ años, rango 19-52. 54 compañeros recibieron tratamiento. 107 pacientes volvieron a la semana y 93 al mes.

RESULTADOS: Clínica: a la semana se consideraron curadas 56 pacientes (52.3\%) y con marcada mejoría 47 (43.9\%); es decir, 103 pacientes (96.3\%) mostraron modificación favorable. Al mes, 72 estaban curadas y 17 mejoraron, lo cual nos da como resultado que 89 de 93 (95.7\%) tuvieron modificación favorable. Hubo fracaso en $3(3.2 \%)$ y recidiva en 1 (1.1\%), cuyo compañero no recibió tratamiento. Microbiología: a la semana se observó erradicación en $72(78.3 \%)$, colonización en $5(5.4 \%)$, y persistencia en 15 (16.3\%). Al mes, erradicación en $76(82.6 \%)$, colonización en $3(3.3 \%)$, persistencia en $11(12.0 \%)$ y reinfección en $2(2.2 \%)$, cuyos compañeros no recibieron terapia. Dos pacientes presentaron eventos adversos que evolucionaron satisfactoriamente.

CONCLUSIONES: 1. El tratamiento de la candidiasis vaginal con $150 \mathrm{mg}$ de fluconazol en dosis única, es una terapia eficaz, de fácil cumplimiento por la paciente y muy bien tolerada. 2. Clínicamente no establece diferencia el hecho que el compañero sexual reciba o no terapia concomitante; sin embargo, microbiológicamente hay diferencia levemente significativa en favor del grupo con tratamiento al compañero.

PALABRAS CLAVES: Candidiasis vaginal, fluconazol.

SUMMARY OBJECTIVE: To assess the efficacy and safety of $150 \mathrm{mg}$ of fluconazole single oral dose for the treatment of vaginal candidiasis.

DESIGN: Prospective, open, non comparative.

SETTING: Outpatient clinics of participating physicians and Ob/Gyn Department of Instituto Materno Infantil of Santafé de Bogotá.

PATIENTS: Outpatients with microbiologically confirmation of vaginal candidiasis (both direc KOH smear and culture).

INTERVENTION: Antifungal treatment with single oral dose, $150 \mathrm{mg}$ of fluconazole. No topical nor systemic antifungal treatment was allowed. At random the stable partner received or did not treatment, so at the end of the study two groups resulted. Controls were done one and four weeks after.

Main outcome measure: cure of fungal infection by negative cultures and disappearance of clinical picture. Effect of treatment of stable sexual partner.

RESULTS: 109 out of 110 patients included were analyzable both for efficacy and safety, because one patient never returned to controls. Mean age: 30.29 \pm 6.6 years, range 19-52. 54 stable sexual partners received treatment, and 55 not. 107 patients returned to one week follow-up and 93 to one month follow-up.

Clinical: at one week 56 out of 107 patients $(52.3 \%)$ were cured and $47(43.9 \%)$ markedly improved; combining both, there was 103 patients $(96.3 \%)$ with favorable modification. At one month, 72 were cured and 17 markedly improved; combining both, 89 out of 93 (95.7\%) showed favorable outcome. Failure occurred in $3(3.2 \%)$ and relapse in $1(1.1 \%)$, sexual partner does not received treatment. Treating partner makes no difference.

Microbiological: at one week, eradication occurred in 72 patients (78.3\%), colonization in $5(5.4 \%)$ and persistence in $15(16.3 \%)$. At one month eradication in $76(82.6 \%)$, colonization in $3(3.3 \%)$, persistence in $11(12.0 \%)$ and relapse in $2(2.2 \%)$, both sexual partners does not received treatment. A small difference in favor of group of partner treatment appeared.

Two patients had adverse events, nausea and dizziness of mild severity that was tolerated in one, and a moderate allergic dermatitis that appeared one day after drug ingestion which required symptomatic treatment in the other. All AE's cleared.

CONCLUSIONS: the treatment of vaginal candidosis with single oral dose of $150 \mathrm{mg}$ of fluconazole is a successful, secure, fast and easy to comply with. KEY WORDS: Vaginal candidiasis, fluconazole.

\footnotetext{
${ }^{1}$ Ginecólogas, Barranquilla, Colombia.

${ }^{2}$ Profesor asistente. Departamento de Obstetricia y Ginecología Universidad Nacional de Colombia. I.M.I. Santafé de Bogotá

${ }^{3}$ Profesor titular Departamento de Obstetricia y Ginecología Universidad del Valle, Cali.
}

${ }^{4}$ Ginecólogo, Clínica Shaio, Santafé de Bogotá, Colombia.
${ }^{5}$ Ginecólogo, ORIENTAME, Santafé de Bogotá, Colombia. 


\section{Introducción}

Reportes americanos informan que aproximadamente 13 millones de vulvoganitis micóticas se presentan anualmente con un incremento del $13 \%$ durante la presente década en población de 18 años y postmenopáusica (1). En Inglaterra las clínicas dedicadas a enfermedades sexualmente transmisibles reportaron un incremento de la candidiasis vaginal de 21.000 casos en 1972 a 38.000 en 1982 (2). La especie Candida fue reportada como la tercera causa como agente patógeno asociado a infecciones nosocomiales sistémicas (3).

Se sabe que la $C$ andida es un saprofito en la naturaleza y se encuentra normalmente en la flora intestinal. Invade los diferentes tejidos en todos aquellos casos de cambio de $\mathrm{pH}$, tratamiento prolongado con antibióticos, casos de inmunodeficiencias, cáncer, diabéticos, embarazo, alcoholismo, obesidad, desnutrición, terapia anticonceptiva entre otros (4). Se presenta como una infección aguda o crónica lesionando boca, bronquios, piel, uñas, vagina y en casos severos septicemia, endocarditis y meningitis (5).

Los síntomas y signos de la candidiasis vaginal son bien conocidos y donde la mayoría de los pacientes consultan por prurito, leucorrea, irritación vulvovaginal y dispareunia. El diagnóstico no puede ser sólo clínico y amerita el cultivo específico para hongos. En un análisis sobre 80 publicaciones en el tema de leucorrea se pudo encontrar porcentajes para Candida albicans, $11.8 \%$ y otros hongos (C. glabrata y C. krusei) $10.8 \%$ (6). La vaginitis por candida es una de las causas frecuentes en la consulta ginecológica, afecta por lo menos una vez en la vida de la mujer a un $75 \%$ de aquellas sexualmente activas y coloniza la vagina de la mujer asintomática en un 15-20\% (7). Se estima su recurrencia en un $10.20 \%(8)$.

El mecanismo de acción de los diferentes compuestos antifúngicos depende de su acción sobre la síntesis del ergosterol, sustancia muy abundante en la candida. La mayoría de los imidazoles (clotrimazol, miconazol, ketoconazol, econazol) y derivados triazólicos (terconazol, itraconazol, fluconazol), derivan su potencia en el efecto que ejercen al inhibir selectivamente la enzima citocromo P-450 dependiente de la 14 alfademetilación del lanosterol (9).

El fluconazol ha demostrado ser activo contra diferentes especies de hongos. Su vida media por vía oral es de 30 horas con niveles en plasma y vagina de $1 \mu \mathrm{g} / \mathrm{g}$ a las 72 horas (10). La concentración mínima inhibitoria MIC para $C$. albicans fue de $0.4-0.8 \mu \mathrm{g} / \mathrm{ml}$, comparable a otros compuestos. Estudios clínicos con dosis única de $150 \mathrm{mg}$ han demostrado tasas de eficacia y seguridad de $84-90 \%$ (11, 12). Fue interés de los autores evaluar la eficacia y seguridad con dosis única de $150 \mathrm{mg}$ de fluconazol en el tratamiento de la candidiasis vaginal sintomática y determinar si la terapia simultánea al compañero sexual juega un papel en la evolución de la enfermedad.

\section{Método}

Por el método abierto se compararon dos grupos de pacientes con candidiasis vaginal sintomática: en un grupo, solamente la paciente recibió tratamiento; en el otro, tanto ella como su compañero sexual recibieron el mismo tratamiento simultáneamente.

A las pacientes que consultaron por flujo $\mathrm{y} / \mathrm{u}$ otros síntomas de vaginitis se les ordenó un frotis vaginal con $\mathrm{KOH}$; si se comprobaba la presencia de hongos (levaduras o micelios) como único patógeno, se propuso su inclusión en el estudio.
Criterios de inclusión: mujeres adultas, ambulatorias, sexualmente activas, con candidiasis vaginal sintomática confirmada por microbiología. Se excluyeron pacientes con intolerancia al fluconazol, en embarazo, en lactancia, y con presencia de otros patógenos o enfermedades concomitantes vaginales o sistémicas que pudieran alterar la interpretación de los resultados clínicos (cáncer, inmunodeprimidas, etc.). También se excluyeron pacientes que recibían o habían recibido terapia antimicótica o antibacteriana durante la semana anterior a su inclusión.

Antes de administrar la dosis única, a la semana y a las cuatro semanas de administrada, se tomaron muestras de secreción vaginal para frotis con $\mathrm{KOH}$ y cultivo de $C$ andida albicans. Igualmente, los signos y síntomas de la infección se valoraron y calificaron antes del tratamiento, y a los 7 y 28 días de administrado. Los compañeros sexuales que recibieron tratamiento fueron seleccionados al azar, siguiendo una tabla aleatoria; no se les abrió historia clínica ni se les hizo examen físico o de laboratorio.

Se obtuvo consentimiento escrito o verbal de todos los pacientes antes de iniciar el tratamiento, siguiendo los lineamientos de la Declaración Revisada de Helsinki (Hong Kong, 1989) y la Ley 23 de 1981 de Colombia sobre Etica Médica.

La Eficacia Clínica del tratamiento fue valorada con base en los siguientes criterios:

Curación: desaparición de los signos y síntomas.

Mejoría: alivio de los signos y síntomas.

Fracaso: ningún cambio en los signos y síntomas o empeoramiento.

La Respuesta Micológica se clasificó así:

Erradicación/Cura: Hallazgos microscópicos negativos y/o ausencia de material cultivable.

Persistencia/Fracaso: Persistencia del patógeno en los frotis y/o cultivo de la lesión.

Colonización: presencia de algunos hongos en pacientes clínicamente curadas que, por consiguiente, no requieren tratamiento; también incluye aquellas pacientes con fresco positivo y cultivos negativos.

Un mes después se valoró, además, la recidiva, considerando como tal la reinfección o recaída después de haberse calificado como curación.

La Seguridad se valoró así:

Excelente: sin efectos secundarios.

Buena: efectos secundarios leves/moderados que desaparecieron espontáneamente.

Regular: efectos secundarios moderados/tolerados, que requirieron tratamiento sintomático.

Mala: eventos severos que requirieron suspender el tratamiento.

Métodos estadísticos: teniendo en cuenta la distribución normal de la población se realizaron análisis descriptivos, como tablas de frecuencias, cálculo de medias y desviaciones estándar, empleando el programa Epiinfo.

\section{Resultados}

Se incluyeron 110 pacientes, de las cuales una nunca retornó a los controles después de recibir el tratamiento. Se conformaron dos grupos al azar: uno (Grupo I) de 54 pacientes a quienes se les entregó tratamiento para el compañero sexual estable y el otro (Grupo II) conformado por 55 pacientes. Al primer control regresaron 107 pacientes y al segundo (cuarta semana) 93.

Las edades oscilaron entre 19 y 52 años, promedio 30.29 \pm 10.58 años (Tabla 1). El peso promedio fue de $56.52 \pm$ 
$7.16 \mathrm{~kg}$ (rango 39-82). La estatura fue de $160.16 \pm 6.42 \mathrm{~cm}$ (rango 150-178).

Tabla 1

DISTRIBUCION POR EDADES

\begin{tabular}{|l|cc|}
\hline $\begin{array}{l}\text { Grupos } \\
\text { De edad }\end{array}$ & \multicolumn{2}{|c|}{ Número de pacientes } \\
\cline { 2 - 3 } & $\begin{array}{c}\text { Con tratamiento } \\
\text { Al compañero }\end{array}$ & $\begin{array}{c}\text { Sin tratamiento } \\
\text { Al compañero }\end{array}$ \\
\hline$<=20$ & 5 & 2 \\
$21-30$ & 23 & 25 \\
$31-40$ & 23 & 23 \\
$41-50$ & 3 & 4 \\
$>50$ & - & 1 \\
\hline Total & 54 & 55 \\
\hline
\end{tabular}

Signos y síntomas: tuvieron una duración promedio de $51.55 \pm 88.42$ días, con un rango entre 1 día y 2 años. En la tabla 2 se puede apreciar la evolución en ambos grupos, siendo notable el predominio del prurito y el flujo antes del tratamiento, y su virtual desaparición al mes de la terapia.

Microbiología: en la tabla 3 se puede observar la evolución tanto en el frotis en fresco $(\mathrm{KOH})$ como en los cultivos a los 7 días y a las 4 semanas de la dosis única de fluconazol.

Eficacia clínica: 107 pacientes de ambos grupos retornaron a los siete días y 93 a las cuatro semanas.

Los resultados obtenidos en el presente estudio son los siguientes:

Tabla $2 \mathrm{~A}$

EVOLUCION DE SINTOMAS POR GRUPO DE TRATAMIENTO

\begin{tabular}{|ccc|}
\hline & $\begin{array}{c}\text { Con tratamiento } \\
\text { Al compañero }\end{array}$ & $\begin{array}{c}\text { Sin tratamiento } \\
\text { Al compañero }\end{array}$ \\
\hline Pretratamiento & $28 / 54$ & $27 / 55$ \\
Disuria 7 días & $6 / 54$ & $6 / 53$ \\
4 semanas & $4 / 46$ & $6 / 47$ \\
\hline Pretratamiento & $52 / 54$ & $52 / 55$ \\
Prurito 7 días & $24 / 54$ & $20 / 53$ \\
4 semanas & $9 / 46$ & $10 / 47$ \\
\hline Pretratamiento & $54 / 54$ & $53 / 55$ \\
Flujo 7 días & $29 / 54$ & $26 / 53$ \\
4 semanas & $14 / 46$ & $16 / 47$ \\
\hline Pretratamiento & $18 / 54$ & $18 / 55$ \\
Mal & & \\
7 días & $5 / 54$ & $4 / 53$ \\
Olor & & \\
4 semanas & $2 / 46$ & $3 / 47$ \\
\hline Pretratamiento & $34 / 54$ & $30 / 55$ \\
Dispareunia 7 días & $10 / 54$ & $13 / 53$ \\
4 semanas & $6 / 46$ & $9 / 47$ \\
\hline
\end{tabular}

Tabla 2B

EVOLUCION DE SIGNOS POR GRUPO DE TRATAMIENTO

\begin{tabular}{|rcc|}
\hline & $\begin{array}{c}\text { Con tratamiento } \\
\text { Al compañero }\end{array}$ & $\begin{array}{c}\text { Sin tratamiento } \\
\text { Al compañero }\end{array}$ \\
\hline Pretratamiento & $45 / 54$ & $44 / 55$ \\
Hiperemia 7 días & $13 / 54$ & $14 / 53$ \\
4 semanas & $3 / 46$ & $10 / 47$ \\
\hline Pretratamiento & $36 / 54$ & $37 / 55$ \\
Muguet 7 días & $10 / 54$ & $11 / 53$ \\
4 semanas & $2 / 46$ & $3 / 47$ \\
\hline
\end{tabular}

Tabla 3

MICROBIOLOGIA POSITIVA PARA CANDIDA ALBICANS

(\%) DE ERRADICACION

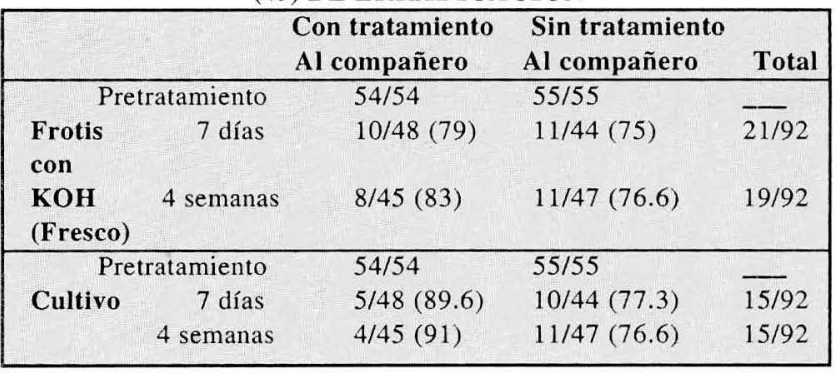

Mantel-Haenzsel estratificado (equivale $\mathrm{CChi}^{2}=5.4 ; \mathrm{p}<0.02$. Limites de Confianza 0.120.86 , en favor del grupo con tratamiento al cónyuge

Curación: a los 7 días desaparecieron todos los signos y síntomas de infección vaginal en $56(52.3 \%)$ de las 107 pacientes que volvieron; a las 4 semanas esta cifra llegó a $72 / 93(77.4 \%)$

Franca mejoría: al $7^{\circ}$ día 47 de las 107 pacientes (43.9\%) mostraron mejoría notoria de sus signos/síntomas; a las 4 semanas sólo $17(18.3 \%)$ de 93 pacientes aún presentaban síntomas.

Fracaso: sólo ocurrió en 4 de las 107 pacientes que retornaron al primer control (3.7\%) y en $3(3.2 \%)$ de 93 al mes.

Recidiva: una (1.1\%) de 93 pacientes a las 4 semanas. El cónyuge de esta paciente no había recibido tratamiento.

En cuanto a evolución clínica, no se apreciaron diferencias significativas entre los 2 grupos comparados.

Eficacia microbiológica: se realizó en 92 pacientes en cada evaluación.

Los datos obtenidos son los siguientes:

Erradicación (desaparición del patógeno pretratamiento) a los 7 días, 72 de 92 pacientes $(78.3 \%)$; a las 4 semanas se incrementó a $76 / 92(82.6 \%)$.

Colonización: (presencia de algunos hongos en pacientes clínicamente curadas que, por consiguiente, no requieren tratamiento; también incluye aquellas pacientes con fresco positivo y cultivos negativos): a los 7 días 5 de 92 $(5.4 \%)$; al mes 3 pacientes (3.3\%).

En la Tabla 5 se observa la sumatoria de Erradicaciones y Colonizaciones, es decir, los resultados microbiológicos favorables en los dos grupos estudiados. Se observa una tendencia favorable en el grupo de los pacientes cuyos compañeros también fueron tratados.

$$
\text { Tabla } 5
$$

RESULTADOS MICROBIOLOGICOS FAVORABLES(ERRADICACION Y COLONIZACION)

\begin{tabular}{|lccc|}
\hline \multicolumn{2}{|c|}{$\begin{array}{c}\text { Con tratamiento } \\
\text { Al compañero }\end{array}$} & $\begin{array}{c}\text { Sin tratamiento } \\
\text { Al compañero }\end{array}$ & Total (\%) \\
\hline 7 días & $43 / 48$ & $34 / 44$ & $77 / 92(83.7)$ \\
4 semanas & $42 / 45$ & $37 / 47$ & $79 / 92(85.8)$ \\
\hline
\end{tabular}

Persistencia (persistencia del patógeno pretratamiento; incluye pacientes con frotis positivo y cultivos positivos): a los 7 días 15 pacientes (16.3\%) y a las cuatro semanas 11 pacientes $(12.0 \%)$.

Recidiva (erradicación del patógeno en el control a los 7 días seguido por su reaparición a las cuatro semanas): 2 
pacientes $(2.2 \%)$. Ninguno de los cónyuges había recibido tratamiento.

Eventos adversos: la seguridad de fluconazol se evaluó sobre las 109 pacientes que retornaron a los controles a los 7 días y cuatro semanas. Sólo se presentaron dos casos de eventos adversos: una paciente presentó náusea y vértigo leves que no requirieron tratamiento. Otra paciente presentó dermatitis alérgica de moderada intensidad que apareció al día siguiente de recibida la droga y requirió tratamiento sintomático. Ambos eventos desaparecieron totalmente.

Excelente (sin eventos adversos): 107 pacientes $(98.2 \%)$.

Buena (eventos leves o tolerables que no requirieron tratamiento): una paciente $(0.9 \%)$.

Regular: (eventos adversos de intensidad leve/moderada que requirieron tratamiento sintomático): una paciente $(0.9 \%)$.

Mala (eventos adversos severos que requirieron tratamiento sintomático): ninguna paciente.

\section{Discusión}

$\mathrm{Al}$ iniciar la búsqueda de candidiasis vaginal tanto clínica como bacteriológica no fue una tarea fácil. La primera conclusión de este trabajo es el engaño que sufre el ginecólogo cuando por las características clásicas descritas en los libros sospecha candidiasis y el laboratorio informa tricomoniasis, exceso de doderlein, flora exaltada. Recomendación general que debemos ser cautos al apresurarnos a diagnosticar candidiasis vaginal macroscópicamente (13).

Con signos y síntomas como prurito, mal olor, leucorrea, disuria, dispareunia, etc., podrá sospecharse candidiasis pero antes hay que evaluar los factores de riesgo de la paciente (embarazo, terapia anticonceptiva, cambios de $\mathrm{pH}$ vaginal) y descartar gérmenes concomitantes en vagina como E. coli, Trichomonas vaginalis, Gardnerella vaginalis, especies de Estafilococos, etc., teniendo en cuenta que pueden asociarse obligándonos a emplear tratamiento combinado (14).

De importancia hoy día el tratamiento de pareja como lo demuestra el presente trabajo y donde se hace necesario concientizar al hombre para que colabore en el tratamiento.

Tabla 6

INCIDENCIA DE VAGINITIS POR C. ALBICANS Y C. NO ALBICANS

\begin{tabular}{|lllll|}
\hline \multicolumn{1}{|c|}{ Especie } & \multicolumn{2}{c|}{$\begin{array}{c}\text { 1970 } \\
\text { 9 estudios }\end{array}$} & \multicolumn{2}{c|}{$\begin{array}{c}\text { 7980 } \\
\text { estudios }\end{array}$} \\
\hline & $\mathrm{N}^{\circ}$ & $\%$ & $\mathrm{~N}^{\circ}$ & $\%$ \\
C. Albicans & 1143 & 90.1 & 573 & 78.7 \\
C. No Albicans & 126 & 9.9 & 155 & 21.3 \\
\hline Total & 1269 & & 728 & \\
\hline
\end{tabular}

Los datos obtenidos del presente trabajo confirman, a pesar de ser una muestra pequeña, la existencia de Candida albicans como patógeno vaginal cuando se decide buscarla. Por laboratorio también se pudo demostrar que, además, se encuentran algunas cepas de Candida no albicans que se excluyeron del trabajo. Por la literatura, se sabe que estas especies de Candida van en aumento como lo demuestran 9 estudios en los años 70 y 7 en los 80 (15). (Tabla 6).

Al comparar nuestros resultados con un multicéntrico realizado en Inglaterra (16) y datos mundiales (11) podemos apreciar en la Tabla 7 que son similares.

Tabla 7

RESULTADOS DE DOS ESTUDIOS ABIERTOS AL AZAR, COMPARATIVOS SOBRE LA EFICACIA DE UNA SOLA DOSIS DE FLUCONAZOL (150 MG/DIA) EN EL TRATAMIENTO DE LA CANDIDIASIS VAGINAL

\begin{tabular}{|c|c|c|c|c|}
\hline País & \multicolumn{2}{|c|}{$\begin{array}{c}\text { Corto plazo } \\
\text { Clínico micológico }\end{array}$} & \multicolumn{2}{|c|}{$\begin{array}{c}\text { Largo plazo } \\
\text { Clínico micológico }\end{array}$} \\
\hline Inglaterra 180 & $\begin{array}{c}142 / 146 \\
97 \%\end{array}$ & $\begin{array}{r}137 / 146 \\
94 \%\end{array}$ & $\begin{array}{l}130 / 147 \\
88 \%\end{array}$ & $\begin{array}{l}109 / 149 \\
73 \%\end{array}$ \\
\hline Colombia 109 & $\begin{array}{r}103 / 107 \\
96.3 \%\end{array}$ & $\begin{array}{l}77 / 92 \\
83.7 \%\end{array}$ & $\begin{array}{l}89 / 93 \\
95.7 \%\end{array}$ & $\begin{array}{l}79 / 92 \\
85.8 \%\end{array}$ \\
\hline Mundial 3080 & $\begin{array}{c}3080 / 3279 \\
94 \%\end{array}$ & $\begin{array}{r}1964 / 2307 \\
85 \%\end{array}$ & $\begin{array}{c}1163 / 1339 \\
87 \%\end{array}$ & $\begin{array}{l}1019 / 1322 \\
77 \%\end{array}$ \\
\hline
\end{tabular}

La mejoría clínica y curación bacteriológica a corto plazo ( 7 días) a esa dosis es buena (96/88\%); a largo plazo (30 días) su eficacia permanece con un porcentaje aceptable y comparativo con un estudio de otra latitud.Las ventajas de la vía oral sobre la local están bien aceptadas dado que el reservorio intestinal está controlado. Las tasas de curación vía orales están en un $84-90 \%$ mientras que con la nistatina es de un $75-80 \%$ (17).

Dentro de los efectos secundarios de la medicación se describen náuseas, molestias gastrointestinales, prurito generalizado. Las estadísticas arrojan cifras 20/244 (8.2\%), $(4 / 100 \%)$ y en el presente estudio $2 / 109(1 \%)$, una con náusea leve que cedió sola y otra con dermatitis que requirió tratamiento sintomático (18-19).

\section{Conclusiones}

Fluconazol en dosis única de $150 \mathrm{mg}$ es una alternativa eficaz, segura, de fácil cumplimiento y cómoda administración en el tratamiento de la candidiasis vaginal. Cuando se trata al compañero sexual estable, los resultados microbiológicos son mejores y parece reducirse la incidencia de recidivas y fracasos.

\section{BIBLIOGRAFIA}

1. Weisberg M. Considerations in therapy for vulvovaginal candidiasis when and whon to treat. in: sobel J. Ed. Clinical perspectives: Terconazol, and advance in volvovaginal candidiasis therapy. lest. ed. New York: McgrawHill, 1988; 2.

2. Bingham JS. Vulvovaginal candidiasis an overview. Acta Derm. Venereol supp. 1986; 121: 39-46.

3. Horan T., Culver D., Javis W. et al. Pathogens causing nosocomial infections. The antimicrobic newletter 1988; 5: 65-67.
4. Hobbs JR., Brigden D. Inmunological aspects of candida vaginitis. Proc. R. Soc. Med. 1977; 70: 11-14.

5. De Sousa HM., Van Uden M. The mode of infection and reinfection in yeast vulvovaginitis. Am. J. Obstet Gynecol. 1960; 80: 1096-1100.

6. Odds FC. Ecology of candida and epidemiology of candidiosis. In candida and candidiosis 2nd Ed. ndon Bailliere Tindall 1989; 93-114.

7. Adler MW., Belsey EM. Sexualy Transmitted diseases in a defined population of woman, Br. Md. J. 1981; 283: 29-32. 
8. Kinghorn GR. Medical overview of vaginal candidiasis. Int. J. Obstet. Gynecol. 1990; 37: 1-8.

9. Grant SM., Clissold SP. Fluconazole. A review of its pharmacodynamic and pharmacokinetic properties, and therapeutic potential in superficial and systemic mycoses. Drugs. 1990; 39(6): 877-916.

10. Houang E. Genital Candidiasis. En Powderly WG and van't Wout JW (eds.) Fluconazole. The antifungal agents, Volume 1. Marius Press, Lancashire, UK, 1992. Ch. 10, 121-132.

11. Rees T., Phillips R. Multicenter comparison of one-day oral therapy with fluconazole or itraconazole in vaginal candidiasis. Int. J. Gynecol Obstet. 1992; 37: 33-38.

12. De los Reyes C., Edelman DE and DeBruin MF. Clinical experience with single-dose fluconazole in vaginal candidiasis. A review of the worldwide database. Int. J. Gynecol Obstet. 1992; 37: 9-15.
13. Per-Anders M. The vaginal ecosytem. Am. J. Obstet. Gynecol. 1991; 165: 1163-1168.

14. Sobel JD. Recurrent vulvovaginal candidiasis N. Engl. J. Med. 1986; 315: 1455-1458.

15. Horowitz BJ. Mycotic vulvovaginitis: A broad overview. Am. J. Obstet. Gynecol. 1991; 165: 1188-1192.

16. Multicenter Study Group. Treatment of candidiasis with a single oral dose of fluconazole. Eur. J. Clin. Microbiol. Infect. Dis. 1988; 7: 364367.

17. Odds FC. Candidiosis of the genitalia. In candida an candidiosis $2 \mathrm{ed}$. Ed. London: Bailliere Tindall. 1988; 124-135.

18. Sobel JD. Fluconazole maintenance therapy in recurrent vulvovaginal candidiasis. Int. J. Gynecol. Obstet. 1992; 37: 17-24.

19. Nixon SA. Vulvovaginitis. The role of patient compliance in treatment success Am J. Obstet. Gynecol. 1991; 165: 1207-1209. 